Proc. 13th International School on Theoretical Physics: Symmetry and Structural Properties of Condensed Matter

\title{
Influence of Photoanode Geometry on Current-Voltage Parameters of the DSSC
}

\author{
P. KWAŚNICKI ${ }^{a, b, *}, J_{\text {. DZIEDZIC }}^{b}$ AND M. INGLOT ${ }^{c}$ \\ ${ }^{a}$ Department of Physical Chemistry and Physicochemical Basis of Environmental Engineering, Institute of \\ Environmental Engineering in Stalowa Wola, John Paul II Catholic University of Lublin, \\ E. Kwiatkowskiego 3A, 37-450 Stalowa Wola, Poland \\ ${ }^{b}$ ML System SA, Zaczernie 190G, 36-062 Zaczernie, Poland \\ ${ }^{c}$ Department of Physics and Medical Engineering, Rzeszów University of Technology, \\ al. Powstańców Warszawy 6, 35-959 Rzeszów, Poland
}

\begin{abstract}
In this work we focus on different geometry of the 3rd generation solar cell in order to verify the influence of the photoanode and electrode shape on the charge transfer between the absorber (dye) and the photoanode and electrode layer. Since dye-sensitized solar cells are still very popular in terms of photovoltaic application especially as being a low cost and transparent solar cell, understanding of the charge transfer mechanism may lead to significant improvement of the efficiency. Research conducted by many scientific communities around the world confirms that such cells have a big advantage over commonly used silicon cells. In addition, the use of a wide range of colours and opportunity to control a transparency creates the possibility of using DSSC as an element integrated with the facade of the building. By laser patterning one can easily change the geometry of the conductive layer such as FTO and modify the charge transfer path in the dye-sensitized solar cell. For this purpose laser ablation process using a semiconductor ytterbium laser $(\lambda=1024 \mathrm{~nm})$ was used. Finally, the current-voltage parameters of DSSC cells with variable geometry of the working electrode were determined using different methods.
\end{abstract}

DOI: 10.12693/APhysPolA.135.1244

PACS/topics: photovoltaics, DSSC, laser ablation, Nyquist graphs

\section{Introduction}

Dye-sensitized solar cell (DSSC) represents the third generation of photovoltaic cells based on organic compounds [1-3]. Dye solar cell contains special chemical compounds that are able to absorb solar radiation and convert them to electricity. The construction of DSSC cells is based on a layered structure consisting of two transparent TCO glass plates, placed parallel to each other and spaced by $\approx 60 \mu \mathrm{m} \mathrm{[4].} \mathrm{One} \mathrm{of} \mathrm{the} \mathrm{plates}$ is coated with a nanocrystalline layer of titanium dioxide covered with an organometallic photosensitive dye (sensitizer) - this system performs the function of photoanodes in the cell $[2,5-7]$. The surface of the second TCO glass plate is usually nanoplatine, constituting a catalytic layer - this system forms a cathode in the cell. The space between the plates is filled with an electrolyte containing the $\mathrm{I}^{-} / \mathrm{I}^{3-}$ redox system. Analysis of literature data indicates the existence of a close dependence between the geometry of the photoelectrode and the efficiency of energy conversion in DSSC [8-10].

\section{Experimental}

Electrochemical impedance spectroscopy (EIS) measurements were made using the AUTOLAB potentiostat

*corresponding author; e-mail: kwasnickipawel@gmail.com
- PGSTAT 302N with frequency response analyzer functions (FRA). Impedance spectra were recorded in the frequency range from $1 \mathrm{MHz}$ to $0.1 \mathrm{~Hz}$ for the alternating current amplitude of $5 \mathrm{mV}$. During the measurement, the cell was excited with light from a solar simulator with an intensity of $\approx 1000 \mathrm{~W} / \mathrm{m}^{2}$.

Optical profilometer results were obtained with the use of optical profilometer PLU Neox Sensofar with the magnification 10× (EPI 10X; $17.5 \mathrm{~mm}$ working distance distance of the lens from the sample).

Solar simulator class AAA, radiation ratio of $1.5 \mathrm{G}$ equipped with halogen lamp of the radiation intensity of $1000 \pm 15 \% \mathrm{~W} / \mathrm{m}^{2}$ with power of $1.43 \mathrm{~kW}$. Short-time instability class $<2 \%$.

\subsection{Sample preparation}

The photovoltaic cells were prepared in the following way: glass substrate with a TCO layer $\left(\mathrm{SnO}_{2}: \mathrm{F}\right)$ with a thickness of $2.2 \mathrm{~mm}$ and a resistivity about $8 \Omega / \mathrm{cm}^{2}$ were treated with laser-structured process using laser with wavelength of $\lambda=1064 \mathrm{~nm}$ to form electrical breaks, thereby separating individual cells from each other. Additionally laser ablation process was also used to pattern the FTO layer of chosen geometry. Important aspect here is that the applied ytterbium laser with high energy and short pulses does not cause microcracks in the glass. The next step was the deposition of $\mathrm{Pt}, \mathrm{TiO}_{2}$, and $\mathrm{Ag}$ layers on a glass substrate using screen printing method. Applied layers were dried, sintered, and fused in a furnace according to given temperature profiles. 
Finally, the $\mathrm{TiO}_{2}$ layer was patterned using laser ablation process and next dyeing process was carried out by a flow method using a ruthenium dye solution.

\section{Results and discussion}

One of the key parameter that proves the degree of conversion of solar to electric energy is the efficiency of the cell, which closely depends on its active surface. In order to correctly determine the efficiency of the cell, its surface was calculated before and after ablation process, based on the results taken with the optical profilometer. Patterns obtained by ablation are illustrated in Fig. 1.

Profilometer results of obtained geometry from which the active area was calculated are depicted in Fig. $2 \mathrm{a}-\mathrm{c}$.
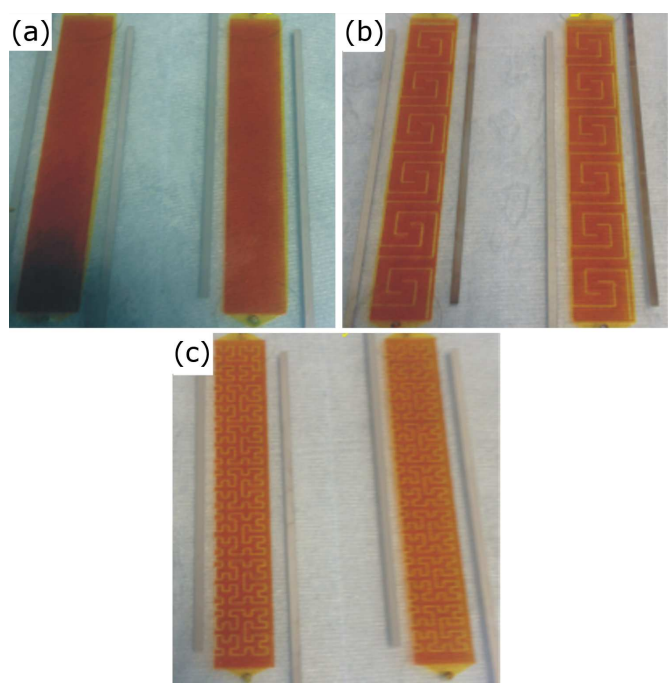

Fig. 1. Patterns obtained by laser ablation process; from left: (a) geometry without ablation, (b) greek column geometry, (c) Hilbert curve geometry.
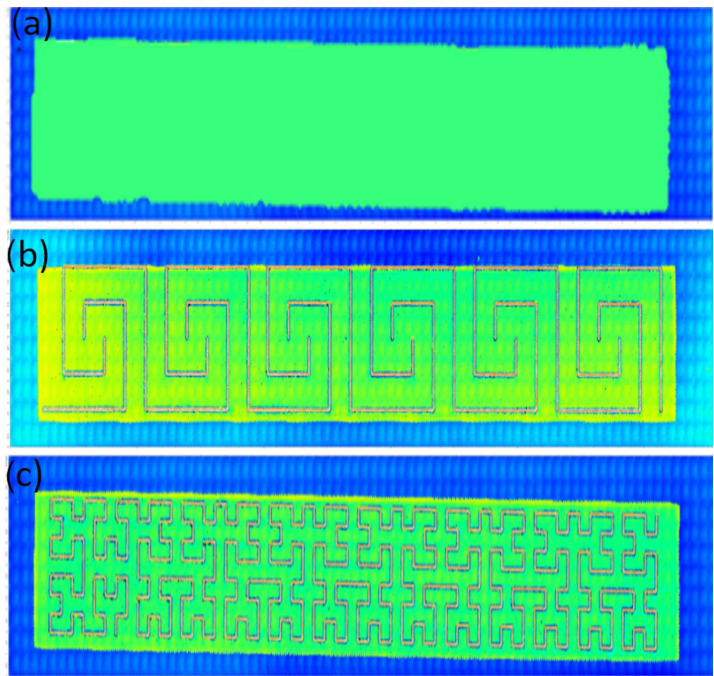

Fig. 2. Profilometer results of obtained geometry: (a) geometry without ablation, (b) Greek column geometry, (c) Hilbert curve geometry.
TABLE I

Calculated active area of a single cell in the DSSC module depending on the geometry

\begin{tabular}{l|c}
\hline \hline Type of geometry & $\begin{array}{c}\text { Active surface } \\
\text { of the cell }\left[\mathrm{cm}^{2}\right]\end{array}$ \\
\hline without ablation & 3.5 \\
Greek column & 2.89 \\
Hilbert curve & 2.7
\end{tabular}

The scoop tool was used to count the pixels in a program designed for processing raster graphics. The obtained results of the calculation of cells active surface depending on the geometry were collected in Table I.

One can clearly see that in case of the cells after ablation process (b) and (c) the active area is significantly lower by around $17 \%$ and $22 \%$, respectively. One can expect the drop of the efficiency by roughly the same values since the dependence of the active area is linear. Next, all the cells were measured using solar simulator in order to find out about the electrical parameters and to check the conversion efficiency. In Figs. 3-5 the results of current-voltage parameters measurements of DSSC cells with variable geometry of the working electrode were collected. Figures show the efficiency Eff, fill factor FF, maximum power point $P_{m}$, short circuit current $I_{s c}$, and open circuit voltage $V_{o c}$ values measured for six different samples.

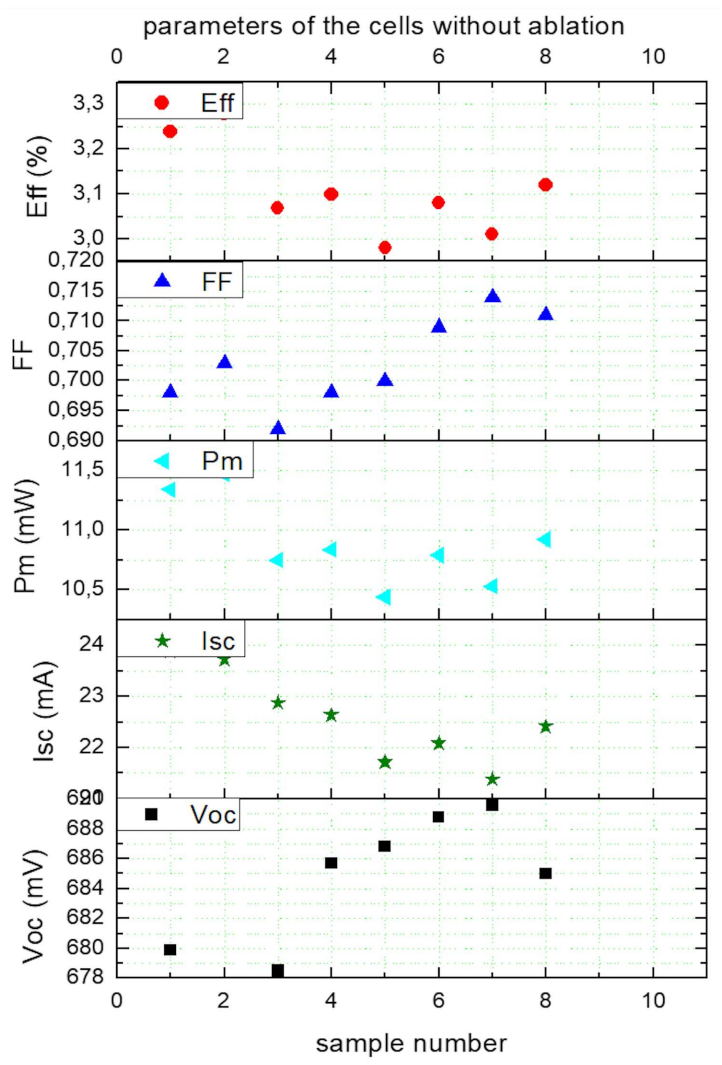

Fig. 3. Measured parameters of the cells without ablation. 


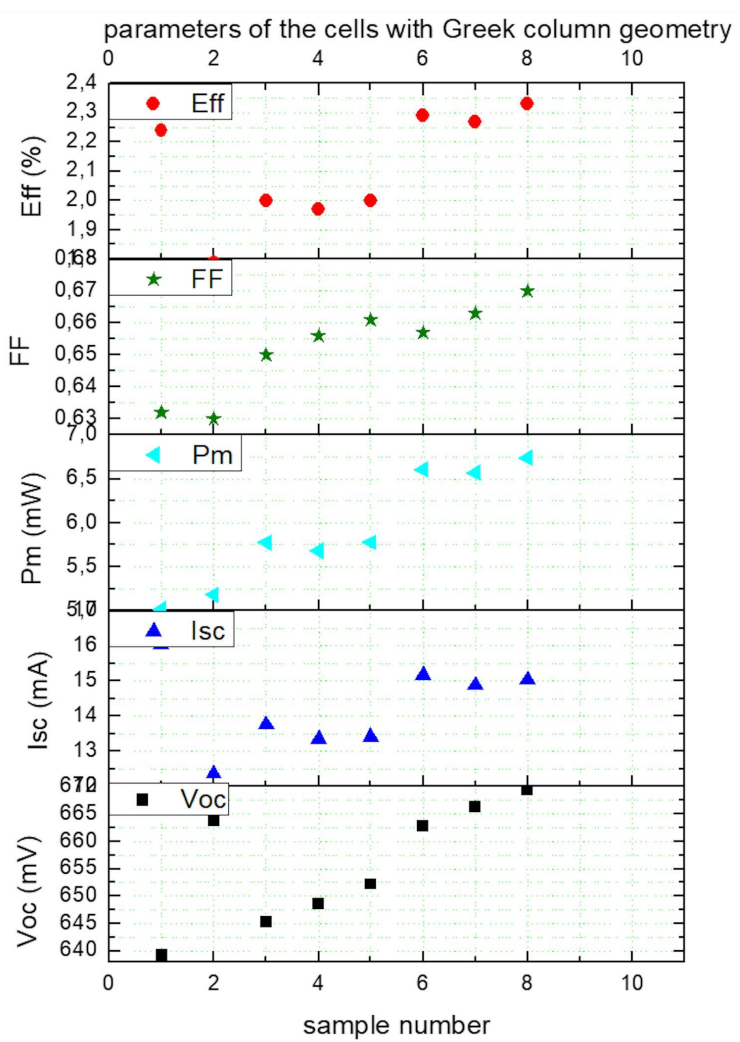

Fig. 4. Measured parameters of the cells with Greek column geometry.

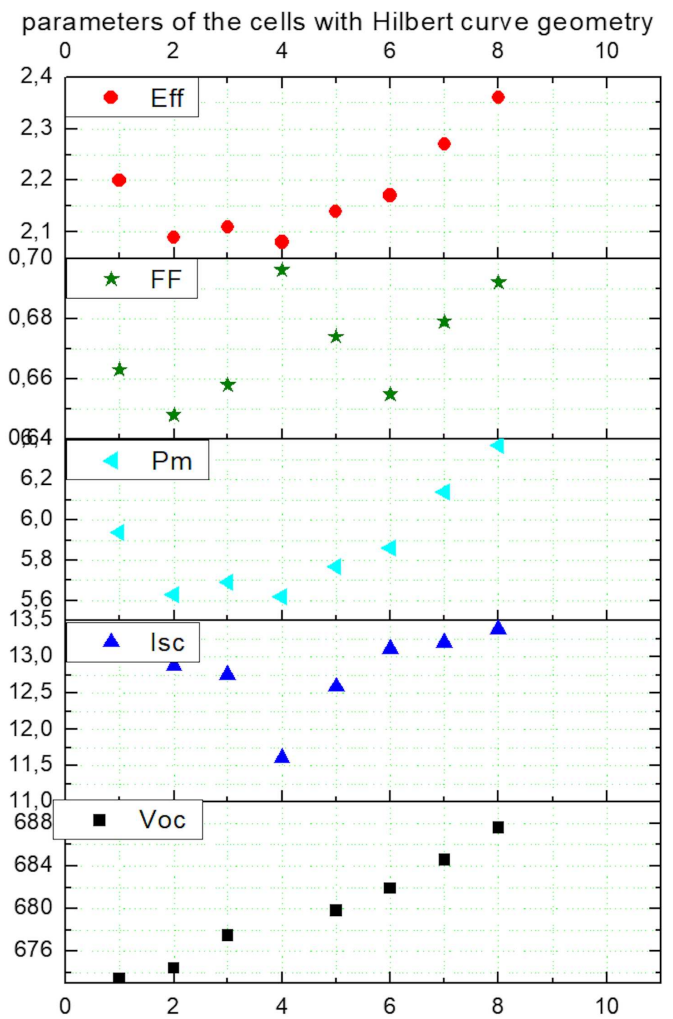

Fig. 5. Measured parameters of the cells with Hilbert curve geometry.
In Fig. 3 the highest value of $V_{o c}, F F$ was observed for sample 7, nonetheless highest conversion efficiency (Eff), $I_{s c}$, and $P_{m}$ for sample 1 . Standard deviation calculated for all the measured parameters shows that that there is not significant dispersion of the value, therefore one can stand that the samples are similar.

In Fig. 4 for the sample with Greek column architecture, one can notice higher dispersion between measured samples, which can be related to the $I_{s c}$ of accuracy of the ablation process. In this case higher value of measured parameters are observed for samples 6-8. Interestingly, all the parameters increase the value.

In Fig. 5 for sample with Hilbert curve architecture, one observes the highest values for sample 8 . Here again we observe quite high dispersion between measured samples comparing it with sample without ablation process.

Table II shows standard deviation for measured parameters from Figs. 3-5.

Standard deviation for measured parame-

TABLE II ters from Figs. 3-5

\begin{tabular}{c|c|c|c}
\hline \hline \multirow{2}{*}{ Parameter } & \multicolumn{3}{|c}{ Standard deviation } \\
\cline { 2 - 4 } & without ablation & Greek column & Hilbert curve \\
\hline$V_{o c}$ & 4.09939 & 11.0253 & 7.32523 \\
$I_{s c}$ & 0.88872 & 1.21476 & 0.5397 \\
$P_{m}$ & 0.35932 & 0.65773 & 0.1883 \\
Eff & 0.10379 & 0.19621 & 0.06768
\end{tabular}

The average value of measured parameters vs. the active area (AA) are shown in Fig. 6. Non-linear dependence on the AA was noticed. The biggest changes were observed for $V_{o c}$ and $F F$.

The results in Figs. 3-6 show that the semiconductor layer $\left(\mathrm{TiO}_{2}\right)$ ablation process has a negative effect on the DSSC cell performance compared to the cell in which the active layer has been printed as a rectangle (cell without ablation; a single $\mathrm{TiO}_{2}$ layer). The main reason of this effect is simply caused by the reduction of the active surface of cell and the difficult flow of charge due to the interruption of the continuity of the active layer. Nonetheless, this effect is not linear and as it is shown in Fig. 6, the efficiency depends not only on the active area but also on the geometry of the layers. One can notice that the highest value is for the samples without ablation process performed, which is rather clear taking into consideration the active area. Although at the same time the lowest active area (sample with the Hilbert curve geometry) has highest value of $V_{o c}, P_{m}$, and efficiency. This fact may be related to decrease of the diffusion length by patterning the conductive layer. Increase in value of FF also may suggest that the ablation process decreases the parasitic resistive losses such as series and shunt resistance. In addition, tests were carried out using EIS for several example geometries of the photoanode layer to check the differences in the electrochemical properties of the individual cells. Measurements were carried out for several cells within DSSC laboratory module. 


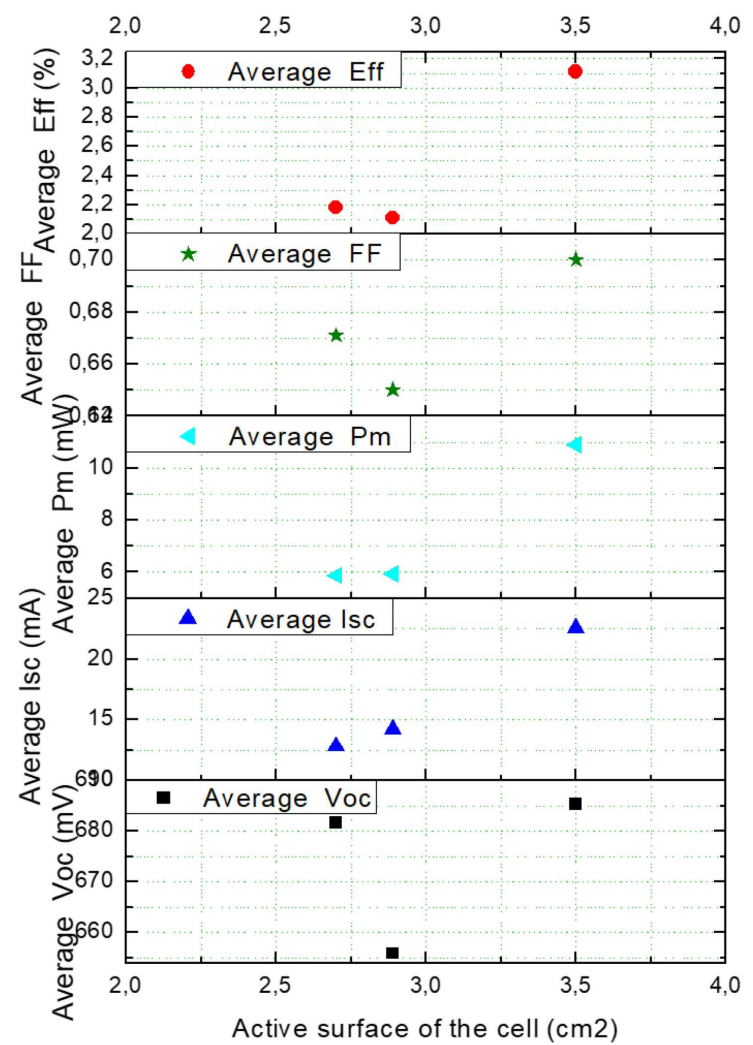

Fig. 6. Average parameters of the cells without ablation $\left(\mathrm{AA}=3.5 \mathrm{~cm}^{2}\right)$, with Greek column geometry $\left(\mathrm{AA}=2.89 \mathrm{~cm}^{2}\right)$ and with Hilbert curve geometry $\left(\mathrm{AA}=2.7 \mathrm{~cm}^{2}\right)$.

Figures 7-9 show the recorded experimental data of the samples along with matching the theoretical curves. Figure 7 showed results of EIS measurement for geometry without ablation, Fig. 8 - the Hilbert curves geometry and Fig. 9 - the Greek column geometry.
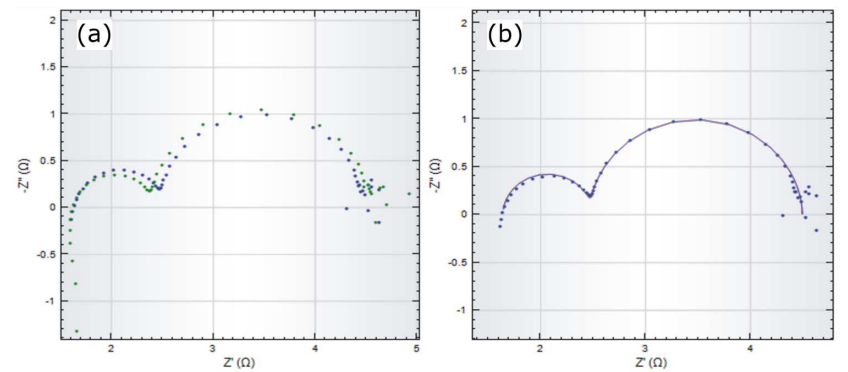

Fig. 7. EIS results for the samples with geometry without ablation: (a) measurement results, (b) measurements and fit results.

From Figs. 6 to 8 one can notice higher influence of the charge transfer process in the metal oxide- electrolyte layers for samples with changed geometry compared with standard sample. This is due to the ablation process and larger interface metal oxide-electrolyte layer.
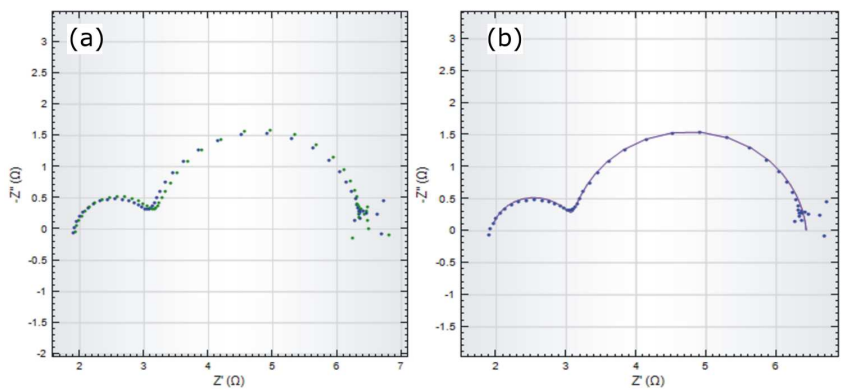

Fig. 8. EIS results for the samples with Hilbert curve geometry: (a) measurement results,(b) measurements and fit results.
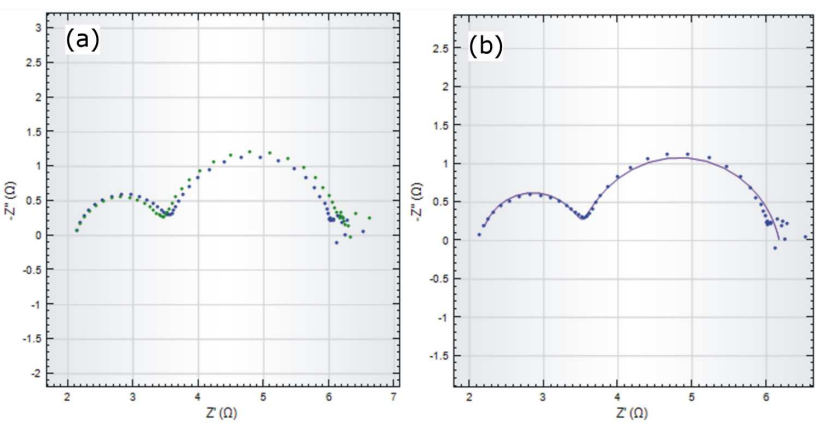

Fig. 9. EIS results for the samples with Greek column geometry: (a) measurement results, (b) measurements and fit results.

TABLE III

A comparison of the values of elements of the Randles electrical circuit resulting from the simulation of fitting to the experimental data of the Nyquist spectra

\begin{tabular}{l|c|c|c|c|c}
\hline \hline $\begin{array}{c}\text { Type of } \\
\text { geometry }\end{array}$ & $R_{s}[\Omega]$ & $R_{1}[\Omega]$ & $R_{2}[\Omega]$ & $\begin{array}{r}C_{P E 1} \\
{[\mathrm{mF}]}\end{array}$ & $\begin{array}{r}C_{P E 2} \\
{[\mathrm{mF}]}\end{array}$ \\
\hline without ablation & 6.67 & 1.43 & 2.66 & 0.039 & 2.85 \\
Hilbert curve & 2.02 & 1.06 & 3.61 & 0.089 & 1.67 \\
Greek column & 1.71 & 0.48 & 2.34 & 0.014 & 3.52
\end{tabular}

Table III summarizes the results of all performed EIS measurements showing the average values of elements of the Randles electrical circuit resulting from the simulation of fitting to the experimental data of the Nyquist spectra.

The obtained Nyquist graphs with impedance spectra reminded two semicircles connected together. To analyse the obtained experimental data, an electric substitute Randles system: $R_{s}\left[\left(R_{1} C_{P E 1}\right)\left(R_{2} C_{P E 2}\right)\right]$ was built. It contains the element of resistance $R_{s}$ corresponding to the series resistance. The $R_{s}$ element is put together with two serially connected circuits:

$$
\begin{aligned}
& \text { 1. } R_{1}, C_{P E 1} \\
& \text { 2. } R_{2}, C_{P E 2}
\end{aligned}
$$

The $C_{P E}$ element has been used due to the dispersion nature of capacitance at the interface, which results from the spatial heterogeneities on the developed electrode surface. 
Resistances $R_{2}$ and $R_{3}$ correspond successively to charge transfer processes on a platinum electrode (respond to the graph in the high frequency range) and charge transfer processes at the photoanode.

The device response in the low frequency range was not obtained, where the Nernst diffusion process in the electrolyte volume takes place (large dispersion of points on the second semicircle of the obtained spectrum). The problem may arise from the duration of the measurement, because the cell under the influence of light excitation obtained temperatures in the range of $22^{\circ} \mathrm{C}$ to $56^{\circ} \mathrm{C}$.

The lowest value of the parasite resistance (in this case $R_{s}$ ) has the cell with the Hilbert curve geometry which is in accordance with the measurements done using solar simulator. Additionally, according to the EIS measurements using laser ablation process one decreases series resistance for both architecture.

\section{Conclusion}

Analysing the results of the $I-V$ and EIS measurements it can be concluded that there is a clear influence of the geometry of the cell on the cell performance especially on the parasite resistance. Furthermore, one can decrease the series resistance in DSSC using laser ablation process, and the $R_{s}$ is related to the geometry. The research clearly confirms that the geometry of photoanode has an impact on energy yields.

\section{Acknowledgments}

This work is supported by The National Centre for Research and Development, grant no. TECHMATSTRATEG1/347431/14/NCBR/2018 received as part of the Strategic Research and Development Program "Modern material technologies" _ "Efficient and light photorechargeable electric energy storage systems based on solar cell-lithium ion battery or solar cell-supercapacitor structures for special applications".

\section{References}

[1] K.S. Elrefi, M.S. Abdel-Latif, T.M. El-Agez, S.A. Taya, Opt. Appl. XLIV, 345 (2014).

[2] A. Ahmed, L. Amadi, S.S. Jenny, Nanosci. Nanoeng. 3, 25 (2015).

[3] S.A. Taya, T.M. El-Agez, T.M. El-Agez, H.S. El-Ghamri, J. Nano- Electron. Phys. 7, 1 (2015).

[4] M. Inglot, J. Dziedzic, Acta Phys. Pol. A 132, 176 (2017).

[5] L. Skowronski, K. Zdunek, K. NowakowskaLangier, R. Chodun, M. Trzcinski, M. Kobierski, M.K. Kustra, A.A. Wachowiak, W. Wachowiak, T. Hiller, A. Grabowski, L. Kurpaska, M.K. Naparty, Surf. Coat. Technol. 282, 16 (2015).

[6] P.M. Ejikeme, A.C. Nwanya, F.I. Ezema, Int. J. Phys. Sci. 6, 5190 (2011).

[7] W.A. Azeez, A.A. Mohammed, A.S.S. Ahmad, Adv Mater. Phys. Chem. 5, 361 (2015).

[8] E. Ramasamy, W.J. Lee, D.Y. Lee, Solar Energy Mater. Solar Cells 93, 1448 (2009).

[9] W. Wang, H. Yuan, D. Xu, J. Xie, X. Chen, Y. He, T. Zhang, Z. Chen, Y. Zhang, H. Shen, J. Photochem. Photobiol. A Chem. 357, 85 (2018).

[10] P. Kwaśnicki, M. Inglot, Acta Phys. Pol. A 132, 179 (2017). 